\title{
La descripción teórica de la detección electroanalítica del fármaco aripiprazol en el medio alcalino, asistida por el oxihidróxido de cobalto trivalente
}

\section{Theoretical description of electroanalytical detection of the drug aripiprazole in alkaline medium assisted by trivalent cobalt oxyhydroxide.}

DOI: $10.46932 / \mathrm{sfjdv2n2-074}$

Received in: March 1st, 2021

Accepted in: May 30th, 2021

Volodymyr V. Tkach

Universidad Nacional de Chernivtsi, 58000, Calle de Kotsyubynsky. 2, Chernivtsi, Ucrania Universidade Federal de Mato Grosso do Sul, Av. Sen. Felinto. Müller, 1555, C/P. 549, 79074-460,

Campo Grande, MS, Brasil

Marta V. Kushnir

Universidad Nacional de Chernivtsi, 58000, Calle de Kotsyubynsky. 2, Chernivtsi, Ucrania

Silvio C. de Oliveira

Universidade Federal de Mato Grosso do Sul, Av. Sen. Felinto. Müller, 1555, C/P. 549, 79074-460,

Campo Grande, MS, Brasil

Adriano O. da Silva

Universidade Federal do Oeste do Pará, Campus Juriti, Rua V. de Souza Andrade, s/n, 68170-000, Juriti, PA,Brasil

Yana G. Ivanushko

Universidad Estatal de Medicina de Bucovina, 58001, Pl. Teatral, 9, Chernivtsi, Ucrania

Olga V. Luganska

UniversidadNacionaldeZaporizhzhia, 69600, CalledeZhukovsky, 66, Zaporizhzhia, Ucrania

Petro I. Yagodynets

1Universidad Nacional de Chernivtsi, 58000, Calle de Kotsyubynsky. 2, Chernivtsi, Ucrania

Zholt O. Kormosh

Universidad Nacional del Este Europeo, 43000, Avda. de la Libertad., 13, Lutsk, Ucrania

Inna M. Dytynchenko

Universidad Estatal de Zhytomyr, 10000, CalleGrande de la Ciudad de Berdychiv, , 40, Zhytomyr, Ucrania

\section{Vitalii V. Lystvan}

Universidad Estatal de Zhytomyr, 10000, CalleGrande de la Ciudad de Berdychiv, , 40, Zhytomyr, Ucrania

\section{Natalia V. Kusyak}

Universidad Estatal de Zhytomyr, 10000, CalleGrande de la Ciudad de Berdychiv, , 40, Zhytomyr, Ucrania 
Correo-e: nightwatcher2401@gmail.com

\title{
RESUMEN
}

En este trabajo se describe, por la primera vez, la posibilidad de la detección electroanalítica del fármaco aripiprazol, asistida por el oxihidróxido de cobalto trivalente. En el medio alcalino, el aripiprazol se hidroliza formando un compuesto hidroquinónico, que se suele oxidar hacia la respectiva quinona y polimerizarse. El análisis del modelo correspondiente confirma que el proceso electroanalítico se mantiene eficiente, aunque sea "ramificado" el mecanismo de oxidación del fármaco. Cuanto a la inestabilidad oscilatoria, esta se realiza por causa de la influencia de la etapa electroquímica a la doble capa eléctrica.

Palabras-clave: aripiprazol, sensor electroquímico, oxihidróxido de cobalto trivalente, oscilaciones electroquímicas, estado estacionario estable.

\begin{abstract}
In this work we describe, for the first time, the possibility of electroanalytical detection of the drug aripiprazole, assisted by trivalent cobalt oxyhydroxide. In the alkaline medium, aripiprazole is hydrolyzed to form a hydroquinone compound, which is usually oxidized to the respective quinone and polymerized. The analysis of the corresponding model confirms that the electroanalytical process remains efficient, although the oxidation mechanism of the drug is "branched". As for the oscillatory instability, it is due to the influence of the electrochemical step on the electrical double layer.
\end{abstract}

Keywords: aripiprazole, electrochemical sensor, trivalent cobalt oxyhydroxide, electrochemical oscillations, steady state.

\section{INTRODUCCIÓN}

Aripiprazol (Fig. 1) es un fármaco antisicótico usado en la amplia variedad de trastornos mentales como esquizofrenia, síndromes bipolares y casos de hiperactividad [ $1-4]$. Se propone, también, su uso para tratamiento de alcoholismo.

Fig. 1. Aripiprazol

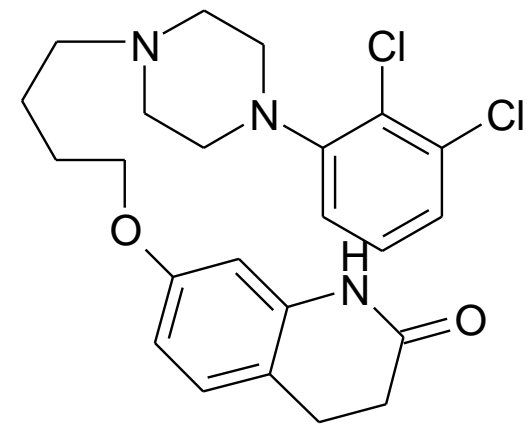

La actividad todos los fármacos se relaciona a la dosis. Aripiprazol no es una excepción, y su exceso se asocia a una multitud de efectos colaterales como bradicardia, parada cardíaca, úlceras, gastroenteritis, gastritis, colitis, entre otros [5 - 10]. Se indica el fármaco con cuidado a personas 
embarazadas y lactantes. Así, el desarrollo de los métodos de la detección de aripiprazol se hace una tárea actual [11 - 12], y los métodos electroanalíticos pretenden serle una solución eficaz [13 - 15].

Poseyendo dos fragmentos aromáticos sustituidos por grupos funcionales donadores y aceptadores de electronos, se puede aripiprazol oxidarse y reducirse química o electroquímicamente. En el primer caso, usándose el medio neutro, la reacción se da mayoritariamente por los fragmentos aromáticos según el mecanismo de polimerización de Díaz [16 - 18], rindiendo productos de media o alta molecularidad.

Ya en el medio alcalino el proceso se hará más dinámico, porque la posibilidad de la hidrólisis de diclorobenceno en hidroquinona disminuirá el potencial de oxidación del producto en relación al fármaco inicial. Otrosí, la trasformación de hidroquinona en quinona también dará su impacto al comportamiento del sistema [19-24].

Para asistir la oxidación del analito, se usan los electrodos químicamente modificados [25 - 32], cuya función es abajar la energía de interacción entre el substrato y ánodo y facilitar la obtención de la señal analítica. Uno de los modificadores de ánodo para la detección de aripiprazol puede ser el oxihidróxido de cobalto, que es un material semiconductor de tipo $p$, sugerido por la parte de los investigadores como alternativa al dióxido de titanio [33 - 35].

Para estabilizar el oxihidróxido de cobalto, que se puede depositar en película o nanopartículas se usa una capa polimérica conductora, que se usa como mediadora de transferencia de electrones. Ambos los procesos oxidativos tienden a acompañarse por inestabilidades electroquímicas [36 - 40], que pueden influenciar el comportamiento del sistema.

Así, el objetivo general de este trabajo es describir teóricamente el comportamiento general del proceso electroanalítico de la detección electroquímica de aripiprazol en medio básico sobre nanopartículas de oxihidróxido de cobalto como sustancia activa. Para tal, nosotros hemos de alcanzar los objetivos específicos a seguir:

- Sugerir el mecanismo más probable de la realización del proceso electroanalítico;

- Desarrollar el modelo, correspondiente al mecanismo;

- Analizando el modelo, derivar las condiciones de las inestabilidades oscilatoria y monotónica, así como de la estabilidad de estado estacionario;

- Comparar el comportamiento del sistema con él de los semejantes [41 - 42].

\section{EL SISTEMA Y SU MODELO}

En medio básico, el cloruro orgánico se hidroliza, rindiendo un compuesto bifenólico, según (1): 

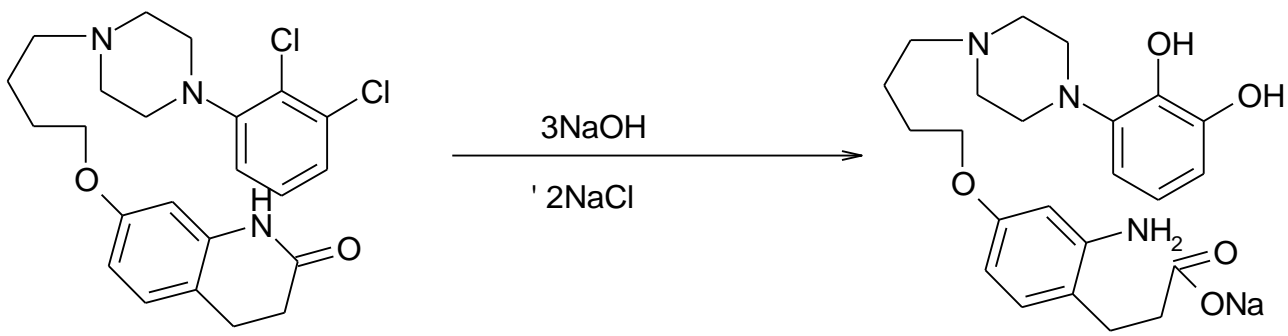

Ambos los anillos aromáticos se hacen más activos después de la reacción (1), lo que abaja el potencial de oxidación del producto en relación al del propio fármaco. Otrosí, se obtienen nuevas formas iónicas, que suelen influenciar la fuerza iónica, la conductividad y la impedancia de la doble capa eléctrica (DCE).

Entre las rutas de oxidación del producto de la reacción (1), las dos principales son la oxidación de hidroquinona en quinona y electropolimerización, que también incluye una interacción intramolecular, análoga a la electropolimerización de la dopamina y algunos tiofenos [16 - 17]. La forma inicial de aripiprazol también interacciona con la cadena polimérica, proveyendo su crecimiento. Así, esquemáticamente, el proceso electroanalítico se hará descrito como en la Fig. 2:

Fig. 2. La representación esquemática del proceso electroanalítico

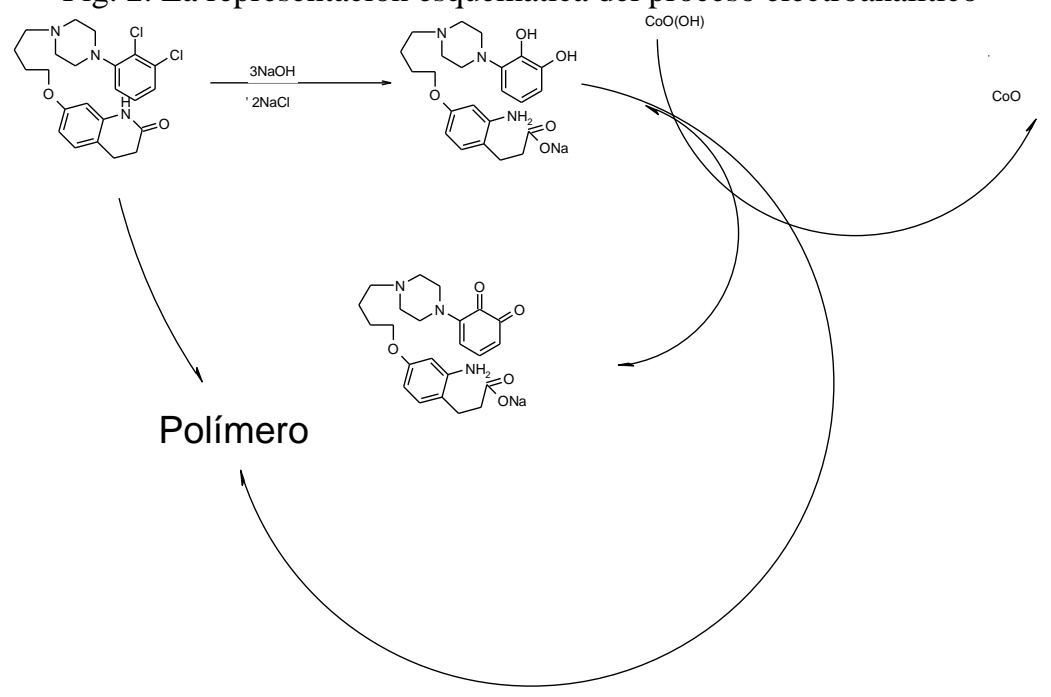

Así, para describir el comportamiento del sistema, nosotros introducimos tres variables:

a - la concentración del aripiprazol en la capa presuperficial;

$\mathrm{a}^{*}$ - la concentración del compuesto bifenólico en la capa presuperficial;

$\mathrm{c}$ - el grado de recubrimiento del área del electrodo por el óxido de cobalto bivalente.

Para simplificar el modelo, suponemos que el reactor se agite intensamente, lo que nos deja menospreciar el flujo de convección. Otrosí, suponemos que el electrólito de soporte esté presente en su 
exceso, dejándonos menospreciar el flujo de migración. Suponemos, también, que el perfil de concentraciones de las sustancias en la capa pre-superficial sea lineal, y su espesor, constante e igual a $\delta$.

Se puede mostrar que el comportamiento del proceso electroanalítico se describe por el conjunto de ecuaciones diferenciales (2):

$$
\left\{\begin{array}{c}
\frac{d a}{d t}=\frac{2}{\delta}\left(\frac{A}{\delta}\left(a_{0}-a\right)-r_{h}-r_{p}\right) \\
\frac{d a *}{d t}=\frac{2}{\delta}\left(r_{h}-r_{q}-r_{p}\right) \\
\frac{d c}{d t}=\frac{1}{C}\left(r_{q}+r_{p}-r_{o}\right)
\end{array}\right.
$$

Aquí, $A$ es el coeficiente de difusión, ao la concentración del aripiprazol en el interior de la solución, C es la concentración máxima superficial del óxido de cobalto, y los parámetros $\mathrm{r}$ son velocidades de las reacciones correspondientes, que se pueden calcular según:

$$
\begin{gathered}
r_{h}=k_{h} a \exp (-b a) \\
r_{q}=k_{q} a *(1-c)^{2} \exp (-b a *) \\
r_{p}=k_{p} a^{* x} a^{y}(1-c)^{z} \exp (-b a *) \exp (-b a) \\
r_{o}=k_{o} c \exp \left(-\frac{F \varphi_{0}}{R T}\right)
\end{gathered}
$$

Aquí, los parámetros $\mathrm{k}$ son constantes de velocidades correspondientes, b es la variable, que describe el impacto de las conversiones de formas iónicas durante las etapas electroquímicas y químicas en la fuerza iónica de la DCE, x, y y z son los órdenes de reacción de electropolimerización asistida, F es el número de Faraday, $\varphi_{0}$ es el salto del potencial, relativo al potencial de carga cero, $\mathrm{R}$ es la constante universal de gases y $\mathrm{T}$ es la temperatura absoluta.

Como se ve, este proceso prevé un impacto intenso a la fuerza iónica de la DCE, que puede llevar a cambios cíclicos de su conductividad y resistencia. Esto podrá provocar el comportamiento oscilatorio, característico para sistemas semejantes [36 - 42]. Sin embargo, el sistema electroanalítico se mantendrá eficiente, como se demostrará abajo.

\section{RESULTADOS Y DISCUSIÓN}

Para investigar el comportamiento del sistema con la detección electroanalítica de aripiprazol, asistida por el oxihidróxido de cobalto, analizamos el conjunto de ecuaciones diferenciales (2) mediante la teoría de estabilidad lineal. Los elementos estacionarios de la matriz funcional de Jacobi para este caso se expondrán como (7): 


$$
\left(\begin{array}{lll}
a_{11} & a_{12} & a_{13} \\
a_{21} & a_{22} & a_{23} \\
a_{31} & a_{32} & a_{33}
\end{array}\right)
$$

en que:

$$
\begin{gathered}
a_{11}=\frac{2}{\delta}\left(-\frac{A}{\delta}-k_{h} \exp (-b a)+b k_{h} a \exp (-b a)-y k_{p} a^{* x} a^{y-1}(1-\right. \\
\left.c)^{z} \exp (-b a *) \exp (-b a)+b k_{p} a^{* x} a^{y}(1-c)^{z} \exp (-b a *) \exp (-b a)\right)(8) \\
a_{12}=\frac{2}{\delta}\left(-x k_{p} a^{* x-1} a^{y}(1-c)^{z} \exp (-b a *) \exp (-b a)+b k_{p} a^{* x} a^{y}(1-c)^{z} \exp (-b a *) \exp (-b a)\right)
\end{gathered}
$$

$$
\begin{gathered}
a_{13}=\frac{2}{\delta}\left(z k_{p} a^{* x} a^{y}(1-c)^{z-1} \exp (-b a *) \exp (-b a)\right)(10) \\
a_{21}=\frac{2}{\delta}\left(k_{h} \exp (-b a)-b k_{h} a \exp (-b a)-y k_{p} a^{* x} a^{y-1}(1-c)^{z} \exp (-b a *) \exp (-b a)+\right. \\
\left.b k_{p} a^{* x} a^{y}(1-c)^{z} \exp (-b a *) \exp (-b a)\right)(11) \\
a_{22}=\frac{2}{\delta}\left(-k_{q}(1-c)^{2} \exp (-b a *)+b k_{q} a *(1-c)^{2} \exp (-b a *)-x k_{p} a^{* x-1} a^{y}(1-\right. \\
\left.c)^{z} \exp (-b a *) \exp (-b a)+b k_{p} a^{* x} a^{y}(1-c)^{z} \exp (-b a *) \exp (-b a)\right)(12) \\
a_{23}=\frac{2}{\delta}\left(2 k_{q} a *(1-c) \exp (-b a *)+z k_{p} a^{* x} a^{y}(1-c)^{z-1} \exp (-b a *) \exp (-b a)\right)(13) \\
a_{31}=\frac{1}{c}\left(-y k_{p} a^{* x} a^{y-1}(1-c)^{z} \exp (-b a *) \exp (-b a)+b k_{p} a^{* x} a^{y}(1-c)^{z} \exp (-b a *) \exp (-b a)\right)
\end{gathered}
$$

$$
\begin{gathered}
a_{32}=\frac{1}{c}\left(k_{q}(1-c)^{2} \exp (-b a *)-b k_{q} a *(1-c)^{2} \exp (-b a *)+x k_{p} a^{* x-1} a^{y}(1-\right. \\
\left.c)^{z} \exp (-b a *) \exp (-b a)-b k_{p} a^{* x} a^{y}(1-c)^{z} \exp (-b a *) \exp (-b a)\right)(15) \\
a_{33}=\frac{1}{c}\left(-2 k_{q} a *(1-c) \exp (-b a *)-z k_{p} a^{* x} a^{y}(1-c)^{z-1} \exp (-b a *) \exp (-b a)-\right. \\
\left.k_{o} \exp \left(-\frac{F \varphi_{0}}{R T}\right)+j k_{o} c \exp \left(-\frac{F \varphi_{0}}{R T}\right)\right)
\end{gathered}
$$

Considerando los elementos de la diagonal principal (8), (12) y (16), nosotros podemos concluir que la inestabilidad oscilatoria en este caso es posible. Además, ella se hará más probable que en los sistemas más simples [41 - 42].

Para que se realice la bifurcación de Hopf, correspondiente al comportamiento oscilatorio, es necesario que haya elementos positivos, correspondientes a la positiva conexión de retorno, en la diagonal principal. Estos elementos son $\quad b k_{h} a \exp (-b a), \quad b k_{q} a *(1-c)^{2} \exp (-b a *)>0, \quad b k_{p} a^{* x} a^{y}(1-$ 
$c)^{z} \exp (-b a *) \exp (-b a)>0$, si $\mathrm{b}>0$ y $j k_{o} c \exp \left(-\frac{F \varphi_{0}}{R T}\right)>0$, si $\mathrm{j}>0$, todos, responsables por la influencia de las etapas químicas y electroquímica a la fuerza iónica de la DCE, que suelen ser cíclicas y, así, correspondientes al comportamiento oscilatorio. Las oscilaciones se prevén frecuentes y de pequeña amplitud.

Cuanto a la estabilidad del estado estacionario, se investiga mediante el criterio Routh-Hurwitz. Evitando las expresiones grandes, introducimos nuevas variables, haciendo que se reescriba el determinante según (15):

$$
\frac{4}{\delta^{2} C}\left|\begin{array}{ccc}
-\kappa-\Sigma-\Xi & -\Lambda & \boldsymbol{Y} \\
\Sigma-\Xi & -P-\Lambda & E+Y \\
\Xi & P+\Lambda & -E-Y-\Omega
\end{array}\right|
$$

Las propiedades del determinante permiten reescribir la expresión (16) como (17)

$$
\frac{4}{\delta^{2} C}\left|\begin{array}{ccc}
-\kappa-\Sigma-\Xi & -\Lambda & Y \\
\Sigma-\Xi & -P-\Lambda & E+Y \\
-\Sigma & 0 & -\Omega
\end{array}\right|
$$

Abriendo los paréntesis y aplicando la condición Det $\mathrm{J}<0$, saliente del criterio, nosotros obtenemos el requisito de estabilidad, expuesto como:

$$
-\Sigma(P Y-\Lambda E)-\Omega(\kappa P+\Sigma P+\Xi P+\kappa \Lambda+2 \Sigma \Lambda)<0
$$

Lo que describe un sistema electroanalitico difusional y cinéticamente controlado. Este requisito está prontamente satisfecho, teniendo el parámetro cinético electroquímico $\Omega$ y químicos $\Sigma$ y $\Xi$ valores positivos, correspondientes a la ausencia o fragilidad de influencias desestabilizadoras. Realmente, si se satisfaga lo arriba mencionado, el valor total del lado izquierdo de la expresión (17) se mueve para valores más negativos. Esto se corresponde a valores más pequeños de deviaciones del estado estacionario, es decir, a su estabilidad.

El proceso electroanalítico es controlado tanto por la difusión, como por la cinética de las reacciones. No habiendo reacciones laterales, capaces de comprometer la estabilidad química de analito y de modificadora fuera del rumbo programado por el mecanismo del proceso, la estabilidad de estado estacionario siempre se corresponderá a la dependencia lineal entre el parámetro electroquímico y la concentración del fármaco, lo que confirma la eficiencia del proceso electroanalítico. 
Ya el límite de detección se corresponde a la inestabilidad monotónica, que define el margen, que separa los estados estacionarios estables de los inestables. Su condición se puede describir como Det J=0, o (18):

$$
-\Sigma(P Y-\Lambda E)-\Omega(\kappa P+\Sigma P+\Xi P+\kappa \Lambda+2 \Sigma \Lambda)=0
$$

Existe, también, la posibilidad de la detección electroanalítica de aripiprazol en medio ácido por vía catódica. En este caso, a depender del pH y modificador de electrodo se puede ocurrir la reducción del grupo amida y deshalogenación del cloruro orgánico. Este caso será descrito en uno de nuestros próximos trabajos.

\section{CONCLUSIONES}

El análisis del sistema con la detección de la acetazolamida sobre el compuesto de poli(5-amino1,4-naftoquinona) con el oxihidróxido de cobalto dejó concluir que se trata de un sistema electroanalítico eficiente. El sistema electroanalítico se controla tanto por la difusión, como por la cinética de las etapas químicas y electroquímica. Se puede fácilmente interpretar la señal electroanalítica, que en la amplia región topológica se corresponde a la dependencia lineal entre la concentración y el parámetro electroquímico. Por otro lado, el comportamiento oscilatorio se hace más probable, por causa de la transformación de un compuesto iónico en la primera etapa química.

\section{CONCLUSIONES}

El análisis del sistema con la detección de la aripiprazol sobre el el oxihidróxido de cobalto dejó concluir que se trata de un sistema electroanalítico eficiente. El sistema electroanalítico se controla tanto por la difusión, como por la cinética de las etapas químicas y electroquímica, por cuenta de trasformaciones de formas iónicas, que dan impacto a DCE. Se puede fácilmente interpretar la señal electroanalítica, que en la amplia región topológica se corresponde a la dependencia lineal entre la concentración y el parámetro electroquímico. Por otro lado, el comportamiento oscilatorio se hace más probable, por causa de la transformación de un compuesto iónico en las etapas químicas. 


\section{REFERENCIAS}

1. B. Crespo-Facorro, M. Ruiz-Veguilla, J. Vázquez-Bourgon et al., Front. Pharmacol., 12(2021) 646701

2. L. D. Crosby, S. Kalanidhi, A. Bonilla et al., J. Transl. Med., 19(2021), 50

3. J. H. Cox, A.E. Cavanna, Exp. Ver. Neurother., 21(2021), 381

4. N. Coustals, M.L. Menard, D. Cohen, J. Child Adolescent Psychopharmacol., 31(2021), 4

5. R. Van der Wall, K. Rotta, K. Ehrhardt et al., Adv. Neurodev. Disorders, 5(2021), 1

6. D.G. Coelho da Silva, L.K. S. Kanazawa, D. dalla Vecchia, Braz. J. Pharm. Sci., 55(2019), 17840

7. S. Mustafa, J. Bougie, M. Miguelez et al., BMC Psych., 19(2019), 114

8. D. Koller, S. Almenara, G. Mejia et al., Adv. Ther., 38(2021), 1054

9. G. Mousalidis, R. Mehboob, B. Papanna et al., Progr. Neur. Psych., 24(2021), 14

10. H Hosseini, N. Zamani, A. Ahmadi, Pharm. Biomed. Res., 6(2020), 169

11. El-Maraghy, H. Salem, Anal. Chem. Lett., 9(2019), 258

12. S.A. Kumbhar, Ch. R. Kokare, B. Shrivastava, H. Choudhury, Curr. Nanomed., 10(2020), 76

13. S. Shin, S. Kim, S. Seo, Transl. Psych., 8(2018), 87

14. S. Kurbanoglu, B. Dogan-Topal, L. Hlavata et al., Electrochim. Acta, 169(2015), 233

15. R. Shrivastava, S. Saxena, S. P. Satsangee et al., Ionics, 21(2015), 2039

16. J. Liebscher, R. Mrowczynski, H. A. Schmidt et al., Langmuir, 29(2013), 10539

17. J. Roncali, Chem. Rev., 92(1992), 711

18. J. Stejskal, M. Kohl, M. Trchova et al., Mater. Adv., 2(2021), 706

19. S. A. B. Bukhari, H. Nasir, L. Pan et al., Sci. Rep., 11(2021), 5044

20. S.S. Ming, N.S.K. Gowthaman, H.N. Lim et al., J. Electroanal. Chem., 883(2021), 115067

21. P-S. Ganesh, G. Shimoga, S-H. Lee et al., J. Anal. Sci. Technol., 12(2021), 20

22. Zh. Lu, Y. Wang, Y. Hasebe, Zh. Zhang, Electroanal., 33(2021), 402

23. K. Chetankumar, B.E. Kumara Swamy, Sensors Int. 1(2020), 100001

24. H. M. Yadav, J-D. Park, H-Ch. Kang, J.J.Lee, Chemosensors, 9(2021), 98 
25. K. Namsheer, Ch. S. Rout, RSC Adv., 11(2021), 5659

26. X. Hong, Y. Liu, Y. Li et al., Polymers, 12(2020), 331

27. Y. Efremenko, V.M. Mirsky, J. Solid State Electrochem., 24(2020), 3105

28. A.S. Agninotri, A. Varghese, M. Nidhin, Appl. Surf. Sci. Adv., 4(2021), 100072

29. V. Sudha, G. Murugadoss, R. Thangamuthu, Sci. Rep., 11(2021), 3413

30. N. Ch. Joshi, Sh. Malik, P. Gururani, Lett. Appl. NanoBioSci., 10(2021), 2339

31. A. Gaiardo, G. Zonta, S. Gherardi et al., Sensors, 20(20), 5910

32. E.H. Sujiono, A.C.M. Said, M.Y. Dahlan et al., J. Nano Electron. Phys., 10(2018), 2034

33. B. Brunetti, D. Elio, J. Food Comp. Anal., 33(2014), 155

34. M.S. Burke, M.G. Kast, L. Trotochaud et. Al., J. Am. Chem. Soc., 137(2015), 3638

35. C. J. Raj, B. Ch. Kim, W. J. Cho et. al., 747(2015), 130

36. O. Stadnik, N. Ivanova, Y. Boldyrev, 218th Int. Electrochem. Soc. Meeting. Abstract \# 2240, http://ma.ecsdl.org/content/MA2010-02/38/2240.full.pdf Accessed at 8th of May 2021

37. Stadnik O. Synthesis, Electrochemical and Photoelectrochemical Properties of the Oxidehydroxide Compounds of Cobalt, Diss. Kand. Chim. N. - Kyiv. - 2011

38. S.U. Rahman, M.S. Ba-Shammakh, Synth. Met. 140(2004), 207.

39. I.Das, N.R.Agrawal, S.A.Ansari, S.K.Gupta,Ind. J. Chem, 47(2008), 1798

40. K. Aoki, I. Mukoyama, J. Chen., Russ. J. Electrochem., 40(2004), 280

41. V. V. Tkach, M. V. Kushnir, V.V. Kopiika et al., Biointerface Res. Appl. Chem., 12(2022), 1273

42. V.V. Tkach, M. V. Kushnir, Ya. G. Ivanushko et al., Appl. J. Env. Eng. Sci., 7(2021), 11 